\title{
Tracking of Blood Pressure in Children and Adolescents in Germany in the Context of Risk Factors for Hypertension
}

\author{
Giselle Sarganas $\left(\mathbb{D},{ }^{1,2}\right.$ Angelika Schaffrath Rosario, ${ }^{1}$ Claudia Niessner, ${ }^{3}$ \\ Alexander Woll, ${ }^{3}$ and Hannelore K. Neuhauser ${ }^{1,2}$ \\ ${ }^{1}$ Robert Koch Institute, Department of Epidemiology and Health Monitoring, Berlin, Germany \\ ${ }^{2}$ DZHK (German Center for Cardiovascular Research), Partner Site Berlin, Germany \\ ${ }^{3}$ Institute for Sports and Sport Science, Karlsruhe Institute of Technology, Karlsruhe, Germany \\ Correspondence should be addressed to Giselle Sarganas; sarganas-margolisg@rki.de
}

Received 1 August 2018; Accepted 10 September 2018; Published 26 September 2018

Academic Editor: Tomohiro Katsuya

Copyright (c) 2018 Giselle Sarganas et al. This is an open access article distributed under the Creative Commons Attribution License, which permits unrestricted use, distribution, and reproduction in any medium, provided the original work is properly cited.

\begin{abstract}
Blood pressure (BP) tracking from childhood to adulthood has two aspects: the ranking stability relative to others over time and the prediction of future values. This study investigates BP tracking in children and adolescents in Germany in the context of hypertension risk factors. BP was measured and analyzed in 2542 participants of the German Health Examination Survey for Children and Adolescents ( $t_{0}$ 2003-2006; 3 to 17-year olds) and of a six year follow-up "Motorik Modul" ( $t_{1}$ 2009-2012; 9 to 24-year olds). BP tracking coefficients were calculated from Spearman's rank-order correlations. Predictive values and logistic regression models were used to forecast $\mathrm{t}_{1}$ - $\mathrm{BP}$ above the hypertension threshold from $\mathrm{t}_{0}$ - $\mathrm{BP}$ as well as from baseline and follow-up hypertension risk factors. BP tracking was moderate (0.33-0.50 for SBP and 0.19-0.39 for DBP) with no statistically significant differences between sex and age groups. Baseline hypertensive BP was the strongest independent predictor of hypertensive BP at follow-up (OR 4.3 and 3.4 for age groups 3-10 and 11-17 years) after adjusting for sex, BMI trajectories, birthweight, parental hypertension, and age-group dependent-sports/physical activity. However, the positive predictive value of baseline hypertensive BP for hypertensive BP at followup in 3- to 10-year olds was only 39\% (34\% in 11- to 17-year olds) and increased only moderately in the presence of additional risk factors. Our analysis with population-based data from Germany shows that BP in children and adolescents tracks only moderately over six years. BP in childhood is the strongest independent predictor of future BP but its predictive value is limited.
\end{abstract}

\section{Introduction}

Blood pressure (BP) elevation is much more common among children and adolescents than previously thought. Moreover, BP in childhood correlates with BP in adulthood [1]; hence children with elevated BP have a higher probability of developing hypertension in adulthood than children with lower BP. However, the usefulness of universal pediatric BP screening is strongly debated due to the only moderate BP tracking correlation from childhood to adulthood [1-4].

BP tracking correlation has two aspects: the stability of $\mathrm{BP}$ ranking relative to others over a specified period of time (calculated as tracking coefficients) and the predictability of future values from baseline values. Meta-analyses have reported moderate tracking coefficients of $0.42-0.38$ for SBP and $0.41-0.28$ for DBP from childhood to adulthood $[1,3]$.
While several studies have investigated BP tracking in children and adolescents and have compared different age groups as well as BP tracking over different time periods, little is known on whether having risk factors for hypertension, such as overweight and obesity, low birthweight, or parental hypertension, is associated with higher BP tracking correlation from childhood to adolescence/young adulthood. Moreover, the predictability of BP measurements from hypertension risk factors in clinically meaningful terms such as positive and negative predictive values has been reported only in the context of screening, not of tracking [5].

Therefore, the aim of this study is twofold: (1) to investigate six year BP tracking correlation coefficients from childhood to adolescence/young adulthood in a large populationbased sample with a broad baseline age range of 3 to 17 years and to illustrate whether this aspect of tracking is stronger 
in subgroups with additional hypertension risk factors; and (2) to analyse the predictability of future BP values above the hypertension threshold from previous BP and hypertension risk factors and to quantify this predictability using different measures of association including odds ratios (OR) and predictive values.

\section{Methods}

2.1. Study Population. The German Health Interview and Examination Survey for Children and Adolescents (KiGGS) is a nationwide study based on a stratified populationregistry sample of 17,641 participants aged $0-17$ years living in Germany (response rate 67\%), including 14,835 participants aged 3-17 years [6]. Details on the baseline and follow-up surveys including its several parallel study modules were published previously [6-8]. At baseline, about half of the participants were invited to take part in the "Motorik Modul Study" (MoMo; response rate 58\%) [7]. The six-year MoMo follow-up had a response rate of $48 \%$ [7]. The present analysis includes data from 2686 children and adolescents who participated in the KiGGS baseline study KiGGS0 ( $\mathrm{t}_{0}$ 20032006; participants aged 3 to 17 years) and the MoMo followup ( $t_{1}$ 2009-2012; participants aged 9 to 24 years) with complete BP data sets and who have not taken antihypertensive medication at $t_{0}$ (unfortunately we do not have information on medication use at $t_{1}$ ). The proportion of complete data sets was higher for those 3-10 years old at $t_{0}$ than for those 11-17 years old. Analyses were stratified by age group: 3-10 years at $t_{0}$ and 9-17 years at $t_{1}$ vs. 11-17 years at $t_{0}$ and 18-24 years at $t_{1}$. We therefore excluded 144 participants who did not fall into these two groups in order to have a clear cutoff in the age group analysis and not mix both hypertension definitions (percentile and $\mathrm{mmHg}$ based; see below), leaving 2542 participants (1269 males and 1273 females) for analysis. Additional follow-up data were available from the telephone interview KiGGS1 2009-2012 [8].

BP measurement methods were standardized and followed the same protocol in KiGGS0 2003-2006 $\left(\mathrm{t}_{0}\right)$ and in the MoMo study 2009-2012 $\left(t_{1}\right)$. At both time-points, BP measurements were taken in the sitting position on a heightadjustable chair with a backrest, the right forearm resting on a table at the level of the heart, the elbow slightly bent, the legs uncrossed, and the feet placed firmly on the floor. Four cuff sizes (cuff bladder in cm $6 \times 12,9 \times 18,12 \times 23$, or $17 \times 38.6)$ were available, which had to cover at least two-thirds of the upper arm length (from the axilla to the antecubital fossa). Two readings of systolic (SBP), diastolic blood pressure (DBP), mean arterial $\mathrm{BP}$, and heart rate were obtained at a two-minute interval with an automated upper arm oscillometric device (Datascope Accutorr Plus, Mahwah, NJ) after a nonstrenuous part of the examination and an additional 5-minute rest in both surveys $[9,10]$. The mean of the 2 systolic and diastolic measurements was used for this analysis.

$\mathrm{BP}$ in children is age, gender, and height dependent; therefore, it is not possible, as for adults, to designate a single BP threshold to define hypertension. BP reference percentiles [11] based on KiGGS data from nonoverweight children were used according to German guidelines [12]: hypertensive BP measurement was SBP or DBP at or above the 95th age-, sex-, and height-specific percentile for children and adolescents under 18 years, or BP $\geq 140 / 90 \mathrm{mmHg}$ irrespective of the percentile. For young adults aged 18 years or older, the absolute cutoff for hypertension $(\geq 140 / 90 \mathrm{mmHg}$ ) was applied. Since percentile-based cutoffs in children and the $140 / 90 \mathrm{mmHg}$ cutoff in adults are not equivalent, analyses were stratified accordingly: baseline ages 3 to 10 years with $t_{1}$-ages 9 to 17 years vs. baseline ages 11 to 17 years with $t_{1}$-ages 18 to 24 years.

In addition, weight and height were measured at $t_{0}$ and $\mathrm{t}_{1}$ according to a common protocol. Weight was measured in underwear to the nearest $0.1 \mathrm{~kg}$ with a calibrated scale (Seca, Birmingham, United Kingdom) according to a standardized protocol [6]. BMI was calculated as the ratio of weight (in $\mathrm{kg}$ ) by height ${ }^{2}$ (in $\mathrm{m}^{2}$ ) and rounded to 3 digits. BMI was categorized as underweight, normal weight, overweight, and obese according to the International Obesity Task Force (IOTF) cutoffs defined by the age-specific percentiles in $<18$ year olds, which at the age 18 and older correspond to the adult cutoff points for overweight $25 \mathrm{~kg} / \mathrm{m}^{2}$ and obesity 30 $\mathrm{kg} / \mathrm{m}^{2}$ [13]. BMI trajectory was defined as the BMI course between $t_{0}$ and $t_{1}$ in 4 categories: "persistently low or normal" defined as under- or normal weight at $\mathrm{t}_{0}$ and at $\mathrm{t}_{1}$; "resolution" as overweight or obese at $t_{0}$ and normal or underweight at $\mathrm{t}_{1}$; "persistently overweight/obesity" (at $\mathrm{t}_{0}$ and at $\mathrm{t}_{1}$ ); and "incident overweight/obesity" as under- or normal weight at $t_{0}$ and overweight or obese at $t_{1}$.

Information on physical activity was collected only in $t_{0}$ : in children aged 3 to 10 years it was assessed through parentreported frequency of sports activities per week (categories: 3 or more, 1-2, and less than once) [14] and in children aged 11 to 17 years through self-reported leisure time physical activities that makes them sweat or get out of breath (categories: $\leq 2.0$, 2.1-4.9, and 5.0-31.0 hours per week) [15].

Birth weight (in $g$ ) was reported by the mother at $t_{0}$; we have classified the variable into less than $2500 \mathrm{~g}$, as low birth weight, or greater than $2500 \mathrm{~g}$.

Parental hypertension was collected at $t_{1}$ with the question: has the mother or father of the study participant ever been diagnosed by a doctor as having hypertension or high blood pressure?

2.2. Statistical Analysis. The analysis was performed in two parts. First, BP tracking was estimated by calculating Spearman's rank-order correlation coefficients stratified by sex and age and potential hypertension risk factors (overweight/obesity, birthweight and parental hypertension). For this purpose, four age groups were chosen: 3 to 6,7 to 10,11 to 14 , and 15 to 17 years. In the first two age groups, the rank order of $t_{0}$-percentiles was correlated with the rank order of $t_{1}$-percentiles. For the last two groups, the rank order of $t_{0}$ percentiles were correlated with the rank order of $t_{1} \mathrm{mmHg}$ values.

For testing statistical differences between correlation coefficients, we have used the software cocor (http://comparingcorrelations.org/) [16].

In the second part of the analysis, we determined the proportion of participants with hypertensive BP measurement 
TABLE 1: Characteristics of the 2542 study participants (1269 males and 1273 females) at baseline (2003-2006) and at follow-up (2009-2012).

\begin{tabular}{|c|c|c|c|c|c|c|}
\hline \multirow[b]{2}{*}{$\begin{array}{l}\text { Characteristics } \\
\text { Age range (years) }\end{array}$} & \multicolumn{3}{|c|}{ Males } & \multicolumn{3}{|c|}{ Females } \\
\hline & $\begin{array}{c}\text { Baseline }\left(\mathrm{t}_{0}\right) \\
(3-17)\end{array}$ & $\begin{array}{c}\text { Follow-up }\left(t_{1}\right) \\
(9-24)\end{array}$ & $\mathrm{p}$-value & $\begin{array}{c}\text { Baseline }\left(t_{0}\right) \\
(3-17)\end{array}$ & $\begin{array}{c}\text { Follow-up }\left(t_{1}\right) \\
(9-24)\end{array}$ & p-value \\
\hline Mean age ${ }^{*}$ & $\begin{array}{c}8.5 \\
(8.3-8.7)\end{array}$ & $\begin{array}{c}14.7 \\
(14.5-14.9)\end{array}$ & 0.000 & $\begin{array}{c}8.5 \\
(8.3-8.7)\end{array}$ & $\begin{array}{c}14.8 \\
(14.6-15.0)\end{array}$ & 0.000 \\
\hline $\mathrm{SBP}(\mathrm{mmHg})^{*}$ & $\begin{array}{c}102.8 \\
(102.2-103.4)\end{array}$ & $\begin{array}{c}117.6 \\
(116.8-118.5)\end{array}$ & 0.000 & $\begin{array}{c}102.8 \\
(102.3-103.4)\end{array}$ & $\begin{array}{c}114.7 \\
(114.0-115.4)\end{array}$ & 0.000 \\
\hline $\mathrm{DBP}(\mathrm{mmHg})^{*}$ & $\begin{array}{c}62.1 \\
(61.7-62.5)\end{array}$ & $\begin{array}{c}66.2 \\
(65.7-66.7)\end{array}$ & 0.001 & $\begin{array}{c}62.7 \\
(62.3-63.1)\end{array}$ & $\begin{array}{c}66.0 \\
(65.5-66.5)\end{array}$ & 0.001 \\
\hline Hypertensive $\mathrm{BP}^{\&}$ & $\begin{array}{c}8.5 \% \\
(7.1-10.2)\end{array}$ & $\begin{array}{c}15.7 \% \\
(13.8-17.8)\end{array}$ & 0.001 & $\begin{array}{c}8.6 \% \\
(7.2-10.3)\end{array}$ & $\begin{array}{c}14.0 \% \\
(12.2-16.0)\end{array}$ & 0.000 \\
\hline $\operatorname{BMI}\left(\mathrm{kg} / \mathrm{m}^{2}\right)^{*}$ & $\begin{array}{c}17.1 \\
(16.9-17.2)\end{array}$ & $\begin{array}{c}20.7 \\
(20.4-20.9)\end{array}$ & 0.000 & $\begin{array}{c}17.1 \\
(17.0-17.3)\end{array}$ & $\begin{array}{c}20.5 \\
(20.3-20.8)\end{array}$ & 0.000 \\
\hline Underweight & $\begin{array}{c}9.8 \% \\
(8.3-11.6)\end{array}$ & $\begin{array}{c}6.1 \% \\
(4.9-7.5)\end{array}$ & 0.001 & $\begin{array}{c}11.3 \% \\
(9.7-13.2)\end{array}$ & $\begin{array}{c}9.4 \% \\
(7.9-11.1)\end{array}$ & 0.104 \\
\hline Normal-weight & $\begin{array}{c}78.9 \% \\
(76.6-81.1)\end{array}$ & $\begin{array}{c}72.5 \% \\
(70.0-74.9)\end{array}$ & 0.000 & $\begin{array}{c}74.0 \% \\
(71.6-76.4)\end{array}$ & $\begin{array}{c}72.6 \% \\
(70.0-75.0)\end{array}$ & 0.372 \\
\hline Overweight & $\begin{array}{c}9.1 \% \\
(7.6-10.8)\end{array}$ & $\begin{array}{c}16.3 \% \\
(14.4-18.5)\end{array}$ & 0.000 & $\begin{array}{c}12.2 \% \\
(10.5-14.1)\end{array}$ & $\begin{array}{c}13.8 \% \\
(12.0-15.8)\end{array}$ & 0.238 \\
\hline Obese & $\begin{array}{c}2.2 \% \\
(1.5-3.2)\end{array}$ & $\begin{array}{c}5.1 \% \\
(4.0-6.5)\end{array}$ & 0.000 & $\begin{array}{c}2.4 \% \\
(1.7-3.4)\end{array}$ & $\begin{array}{c}4.3 \% \\
(3.3-5.5)\end{array}$ & 0.011 \\
\hline $\begin{array}{l}\text { Sports activities ( } 3 \text { or more } \\
\text { times/week) ( } 3-10 \text { years) }\end{array}$ & $\begin{array}{c}43.2 \% \\
(40.1-46.4)\end{array}$ & - & - & $\begin{array}{c}35.5 \% \\
(32.4-38.6)\end{array}$ & - & - \\
\hline $\begin{array}{l}\text { Sports activities }(\mathrm{h} / \text { week })^{*} \\
(11-17 \text { years })\end{array}$ & $\begin{array}{c}7.6 \\
(6.5-8.8)\end{array}$ & - & - & $\begin{array}{c}5.2 \\
(4.7-5.7)\end{array}$ & - & - \\
\hline Parental hypertension & - & $\begin{array}{c}28.1 \% \\
(25.7-30.8)\end{array}$ & - & - & $\begin{array}{c}30.3 \% \\
(27.7-32.9)\end{array}$ & - \\
\hline $\begin{array}{l}\text { Low birthweight } \\
(<2500 \mathrm{~g})\end{array}$ & $\begin{array}{c}5.0 \% \\
(3.9-6.4)\end{array}$ & - & - & $\begin{array}{c}6.3 \% \\
(5.1-7.8)\end{array}$ & - & - \\
\hline
\end{tabular}

* mean $(95 \% \mathrm{CI})$.

${ }^{\&}$ SBP or DBP at or above the 95th age-, sex-, and height-specific percentile for children and adolescents under 18 years, or BP $\geq 140 / 90$ mmHg irrespective of the percentile. For young adults aged 18 years or older, the absolute cutoffs for hypertension $(\geq 140 / 90 \mathrm{mmHg})$ were applied.

at $t_{0}$ and $t_{1}$. For calculating the positive predictive value (PPV) of hypertensive BP at $\mathrm{t}_{0}$ for future hypertensive BP (six years later at $t_{1}$ ), as well as the negative predictive value (NPV) of normotensive $\mathrm{BP}$ at $\mathrm{t}_{0}$ for future normotensive BP, we used the software MedCalc (https://www.medcalc.org/ calc/diagnostic_test.php). We calculated the PPV and NPV also for risk factor combinations, e.g., for having hypertensive $\mathrm{BP}$ and being obese at $\mathrm{t}_{0}$. In sensitivity analyses, we checked whether the results were altered when using weighting factors in the analysis. These weights take selective participation in the MoMo study and the drop-out between $t_{0}$ and $t_{1}$ into account. They are the inverse of the probability to participate in the MoMo study/the follow-up, estimated in a logistic regression model including several relevant KiGGS variables as predictors. Since no weights are available for the exact population under study here, we used three existing sets of weights: longitudinal weights adjusting the KiGGS followup to the KiGGS baseline, longitudinal weights adjusting the MoMo follow-up to the MoMo baseline, and cross-sectional MoMo weights adjusting the MoMo follow-up to the KiGGS1 follow-up.

Furthermore, we investigated the prediction of hypertensive $t_{1}$-BP from $t_{0}$-BP and other baseline and follow-up risk factors for hypertension, using logistic regression models.
Models were run separately for the $t_{0}$-age-group 3 to 10 years (percentile-defined $t_{1}$-hypertension) and the $t_{0}$-age-group 11 to 17 years (140/90 $\mathrm{mmHg}$ threshold $\mathrm{t}_{1}$-hypertension). STATA SE14.1 was used for the analyses.

\section{Results}

The characteristics of the 2542 study participants (1269 males and 1273 females) at baseline (2003-2006) and six year followup (2009-2012) are presented in Table 1. At six-year followup, mean SBP had significantly increased by $14.8 \mathrm{mmHg}$ in males and by $11.9 \mathrm{mmHg}$ in females. In addition, the proportion of overweight in males and obesity in both sexes was at follow-up statistically significantly greater compared to baseline (Table 1).

Spearman rank-order correlations for BP tracking from childhood to adolescence/young adulthood are shown in Table 2. Tracking coefficients were higher for SBP (ranging for sex-age groups from 0.33 to 0.50 ) than for DBP (ranging for sex-age groups from 0.19 to 0.39 ). Although tracking coefficients increased with age in males, this increase was not statistically significant. Other differences between sex and age groups were as well not statistically significant. 
TABLE 2: Spearman rank-order correlation coefficients for six year-tracking of BP in children and adolescents in Germany.

\begin{tabular}{|c|c|c|c|}
\hline \multirow{2}{*}{ Baseline age } & \multirow{2}{*}{$\mathrm{n}$} & \multicolumn{2}{|c|}{ Tracking coefficients } \\
\hline & & SBP & DBP \\
\hline \multicolumn{4}{|l|}{$3-6$ years $^{1}$} \\
\hline All & 1063 & 0.38 & 0.24 \\
\hline Male & 530 & 0.33 & 0.19 \\
\hline Female & 533 & 0.44 & 0.30 \\
\hline \multicolumn{4}{|l|}{$7-10$ years $^{1}$} \\
\hline All & 953 & 0.42 & 0.36 \\
\hline Male & 481 & 0.41 & 0.30 \\
\hline Female & 472 & 0.42 & 0.42 \\
\hline \multicolumn{4}{|l|}{$11-14$ years $^{2}$} \\
\hline All & 320 & 0.34 & 0.34 \\
\hline Male & 155 & 0.43 & 0.32 \\
\hline Female & 165 & 0.36 & 0.38 \\
\hline \multicolumn{4}{|l|}{$15-17$ years $^{2}$} \\
\hline All & 206 & 0.47 & 0.36 \\
\hline Male & 103 & 0.50 & 0.34 \\
\hline Female & 103 & 0.46 & 0.39 \\
\hline
\end{tabular}

${ }^{1} \mathrm{t}_{0}-\mathrm{BP}$ and $\mathrm{t}_{1}-\mathrm{BP}$ in age-, sex-, and height-specific percentiles; ${ }^{2} \mathrm{t}_{0}-\mathrm{BP}$ in percentiles and $\mathrm{t}_{1}-\mathrm{BP}$ in $\mathrm{mmHg}$.

All Spearman rank-order $;$ sup/iccorrelation coefficients were statistically significant at a p-value $<0.001$.

The BP tracking coefficients stratified by birthweight, parental hypertension and BMI trajectories remained in the order of magnitude of the tracking coefficients for the overall sex-age groups; i.e., children with these hypertension risk factors did not have substantially higher SBP or DBP tracking correlation coefficients compared to children without these risk factors (data not shown).

We investigated the prevalence of hypertensive BP measurement at baseline and at follow-up in a younger age group (baseline age 3 to 10 years, pediatric percentile-based definition of hypertensive $\mathrm{BP}$ at $\mathrm{t}_{1}$ ) and an older age group (baseline age 11 to 17 years, adult definition of hypertensive $\mathrm{BP}$ at $\mathrm{t}_{1}$ ) (Figure 1). Among children aged 3 to 10 years, $7.9 \%$ (95\% CI 6.7-9.1) had hypertensive BP at baseline. Of these, $39 \%$ ( $n=62$ out of 159 ) also had hypertensive BP at followup. The proportion of children with persistent hypertensive BP (at $t_{0}$ and $t_{1}$ ) accounted for $3.1 \%$ of this age group; incident hypertensive $\mathrm{BP}$ (children with normal BP at $\mathrm{t}_{0}$ and hypertensive BP measurement at $t_{1}$ ) was observed in $11.6 \%$; resolution of hypertensive $\mathrm{BP}$ (children with hypertensive $\mathrm{BP}$ at $\mathrm{t}_{0}$ and a normal BP at $\mathrm{t}_{1}$ ) was observed in $4.8 \%$ and the proportion of persistently normal BP in the 3- to 10-year-old group accounted for $80.5 \%$. Among children and adolescents aged 11 to 17 years, $11.2 \%$ (95\% CI 8.6-14) had hypertensive BP at baseline. Of these, $34 \%(n=20$ out of 59$)$ also had hypertensive BP at follow-up. In this age group, persistent hypertensive BP accounted for 3.8\%; incident hypertensive $\mathrm{BP}$ was observed in $12 \%$; resolution of hypertensive BP in $7.4 \%$ and persistently normal BP in $77 \%$ of the study population (Figure 1).

The univariate and multivariable logistic regression (Table 3) showed the factors associated with having hypertensive $\mathrm{BP}$ at $\mathrm{t}_{1}$. In the models for both the younger and the older age group hypertensive $\mathrm{BP}$ at $\mathrm{t}_{0}$ was one of the major determinants (OR 4.25 (95\% CI 2.90-6.23) and OR 3.35 (95\% CI 1.60-7.01)). Further, increasing age was also identified as a risk factor for hypertensive BP at follow-up in both age groups. In the 3 - to 10 -year-old group, children with a persistently overweight/obesity as well as those with an incident overweight/obesity were more likely to have hypertensive $\mathrm{BP}$ at $\mathrm{t}_{1}$ (OR 2.70 (95\% CI 1.78-4.12) and OR $4.44(95 \%$ CI 3.08-6.42), respectively) compared to children who had a persistently low or normal BMI. A gender difference was found only in the 11- to 17-year-old group, in which females were less likely to have hypertensive BP at $\mathrm{t}_{1}$ compared with their male peers. Moreover, only in the 11- to 17-year-old group, parental hypertension was found to be a determinant for hypertensive BP at follow-up.

The probability of having hypertensive BP at six year follow-up increased with baseline BP percentile (Figure 2) but did not exceed $50 \%$ with the exception of a small subgroup ( $\mathrm{n}$ $=8$ ) of 11- to 17-year-old males having a SBP between 90th and 94.9th percentile (prehypertensive at $t_{0}$ ) with a probability of $88 \%$ (95\% CI 47-99.7) of having hypertensive BP at $t_{1}$.

The positive (PPV) and negative predictive values (NPV) according to age at baseline and risk factors are presented in Table 4. The PPV of baseline hypertensive BP for hypertensive BP six years later was 39\% (95\% CI 32-46) for 3- to 10-yearold children and 34\% (95\% CI 24-45) for 11- to 17-year olds. The NPV of non-hypertensive baseline BP was $87 \%(95 \%$ CI $87-88)$ and $87 \%$ (95\% CI 85-88) respectively. In the 3 to 10 -year-old group, the PPV increased as risk factors were combined: for instance, $53 \%$ (95\% CI 30-76) of 3- to 10-yearold children with a hypertensive BP and obesity at baseline also had hypertensive BP six years later. In the 11- to 17-yearold group, similar probabilities of having hypertensive BP at 
TABLE 3: Predictors of hypertensive $\mathrm{BP}^{*}$ at six-year follow-up in children and adolescents in Germany.

\begin{tabular}{|c|c|c|c|c|}
\hline \multirow[b]{2}{*}{ 3- to 10-year-olds (at baseline) } & \multicolumn{2}{|c|}{ univariable analysis } & \multicolumn{2}{|c|}{ multivariable analysis } \\
\hline & OR $(95 \% \mathrm{CI})$ & p-value & OR $(95 \% \mathrm{CI})$ & p-value \\
\hline \multicolumn{5}{|l|}{ Sex } \\
\hline Male & Ref & 0.50 & Ref & 0.19 \\
\hline Female & $1.09(0.85-1.39)$ & & $1.21(0.91-1.59)$ & \\
\hline Age & $1.14(1.08-1.21)$ & $<0.001$ & $1.15(1.08-1.23)$ & $<0.001$ \\
\hline \multicolumn{5}{|l|}{$\mathrm{BP} \mathrm{t}_{0}$} \\
\hline normal $(<\mathrm{P} 95)$ & Ref & $<0.001$ & Ref & $<0.001$ \\
\hline hypertensive ( $\geq$ P95) & $4.44(3.14-6.28)$ & & $4.25(2.90-6.23)$ & \\
\hline \multicolumn{5}{|l|}{ BMI trajectory $\left(\mathrm{t}_{0}\right.$ to $\left.\mathrm{t}_{1}\right)$} \\
\hline persistently low or normal & Ref & $<0.001$ & Ref & $<0.001$ \\
\hline resolution & $1.74(0.96-3.16)$ & & $1.64(0.86-3.11)$ & \\
\hline persistently overweight/obesity & $3.87(2.67-5.61)$ & & $2.70(1.78-4.12)$ & \\
\hline incident overweight/obesity & $3.95(2.83-5.51)$ & & $4.44(3.08-6.42)$ & \\
\hline \multicolumn{5}{|l|}{ Sport activities (times/week) } \\
\hline$<1$ & Ref & 0.14 & Ref & 0.07 \\
\hline $1-2$ & $1.21(0.85-1.73)$ & & $1.30(0.87-1.92)$ & \\
\hline$\geq 3$ & $1.42(0.99-2.02)$ & & $1.58(1.06-2.35)$ & \\
\hline \multicolumn{5}{|l|}{ Parental hypertension } \\
\hline No & Ref & 0.02 & Ref & 0.48 \\
\hline Yes & $1.38(1.06-1.80)$ & & $1.11(0.83-1.49)$ & \\
\hline \multicolumn{5}{|l|}{ Low birthweight $(<2500 \mathrm{~g})$} \\
\hline No & Ref & 0.59 & Ref & 0.69 \\
\hline Yes & $1.14(0.70-1.88)$ & & $1.12(0.64-1.96)$ & \\
\hline \multicolumn{5}{|l|}{ 11- to 17-year-olds (at baseline) } \\
\hline \multicolumn{5}{|l|}{ Sex } \\
\hline Male & Ref & $<0.001$ & Ref & $<0.001$ \\
\hline Female & $0.37(0.22-0.61)$ & & $0.33(0.18-0.61)$ & \\
\hline Age & $1.22(1.07-1.40)$ & $<0.01$ & $1.21(1.03-1.42)$ & 0.02 \\
\hline \multicolumn{5}{|l|}{$\mathrm{BP} \mathrm{t}_{0}$} \\
\hline normal $(<\mathrm{P} 95)$ & Ref & $<0.001$ & Ref & 0.001 \\
\hline hypertensive ( $\geq$ P95) & $3.41(1.87-6.23)$ & & $3.35(1.60-7.01)$ & \\
\hline \multicolumn{5}{|l|}{ BMI trajectory $\left(t_{0}\right.$ to $\left.t_{1}\right)$} \\
\hline persistently low or normal & Ref & 0.07 & Ref & 0.32 \\
\hline resolution & $1.82(0.58-5.70)$ & & $0.85(0.17-4.19)$ & \\
\hline persistently overweight/obesity & $2.23(1.19-4.16)$ & & $1.96(0.89-4.29)$ & \\
\hline incident overweight/obesity & $1.50(0.73-3.08)$ & & $1.59(0.65-3.88)$ & \\
\hline \multicolumn{5}{|l|}{ Physical activities (hours/week) } \\
\hline$\leq 2.0$ & Ref & 0.24 & Ref & 0.42 \\
\hline $2.1-4.9$ & $1.76(0.76-4.07)$ & & $1.75(0.69-4.40)$ & \\
\hline $5.0-31.0$ & $1.93(0.90-4.13)$ & & $1.70(0.74-3.91)$ & \\
\hline \multicolumn{5}{|l|}{ Parental hypertension } \\
\hline No & Ref & 0.03 & Ref & 0.04 \\
\hline Yes & $1.78(1.07-2.95)$ & & $1.85(1.02-3.33)$ & \\
\hline \multicolumn{5}{|l|}{ Low birthweight $(<2500 \mathrm{~g})$} \\
\hline No & Ref & 0.46 & Ref & 0.56 \\
\hline Yes & $0.57(0.13-2.50)$ & & $0.62(0.12-3.16)$ & \\
\hline
\end{tabular}




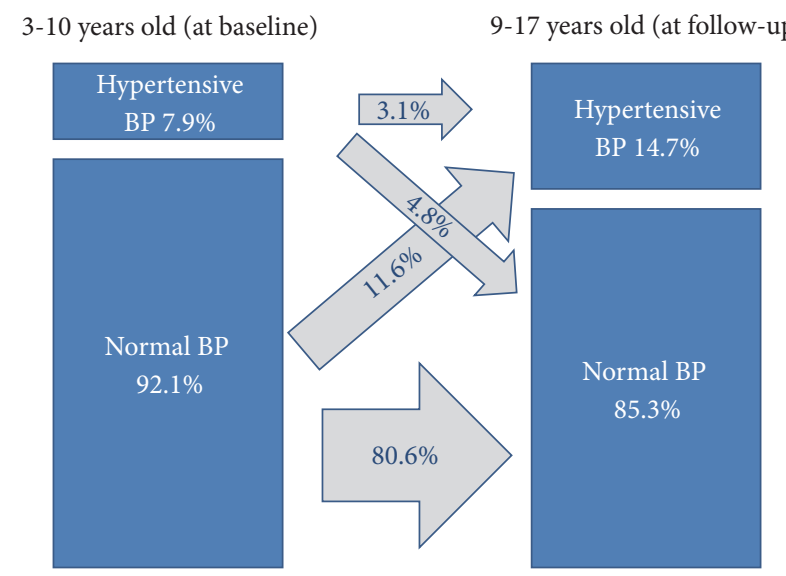

Hypertensive BP: SBP or DBP $\geq 95^{\text {th }}$ percentile or $\geq 140 / 90 \mathrm{mmHg}$

(a) Distribution of 2016 study participants at baseline (2003-2006) and at follow-up (2009-2012) according to the BP measures

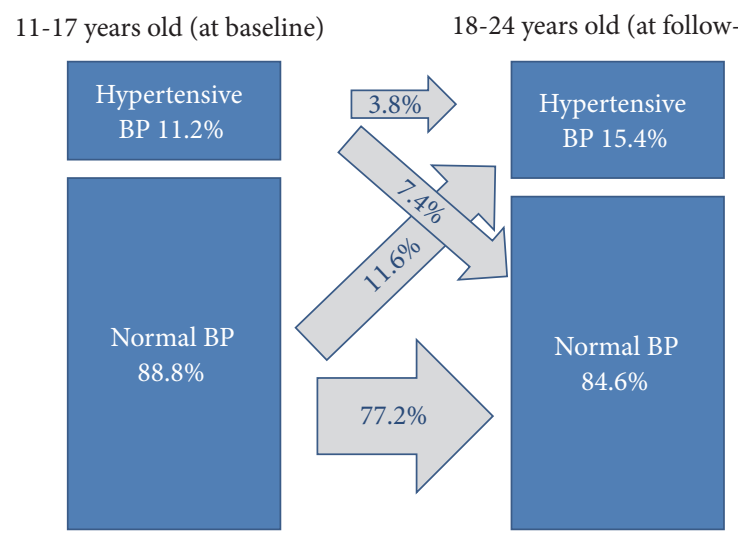

Hypertensive BP at $t_{0}$ : SBP or DBP $\geq 95^{\text {th }}$ percentile or $\geq 140 / 90 \mathrm{mmHg}$ Hypertensive BP at $\mathrm{t}_{1}(\geq 18$ year olds): $\geq 140 / 90 \mathrm{mmHg}$

(b) Distribution of 526 study participants at baseline (2003-2006) and at follow-up (2009-2012) according to the BP measures

FIGURE 1: Blood pressure in 2542 study participants at baseline (2003-2006) and at follow-up (2009-2012).

follow-up were observed in obese children at baseline (50\%; 95\% CI 27-74) and children with a hypertensive BP at baseline and parental hypertension (48\%; 95\% CI 30-67) (Table 4).

\section{Discussion}

This study investigates different aspects of BP tracking from childhood to adolescence/young adulthood with recent population-based data from Germany. Applying various metrics for assessing tracking including correlation coefficients, positive predictive values, and ORs from multivariable predictive modelling, BP tracking from childhood to adolescence and young adulthood was generally moderate. Our results confirm previous findings that BP in childhood and adolescence is one of the strongest predictors of future $\mathrm{BP}$; however, it is one of the strongest predictors among several moderate or weak predictors. Among children and adolescents aged 3 to 17 years with hypertensive BP at baseline (mean of two measurements taken on one occasion), about one in three had hypertensive BP six years later. In population subgroups of children with additional hypertension risk factors such as overweight or parental hypertension, the PPV of baseline hypertensive BP increased but was not much higher than $50 \%$.

In line with previous findings, we found moderate BP tracking coefficients, higher for SBP than for DBP [1-3]. Stratified by age and sex, we found a SBP tracking correlation in the range of 0.33 to 0.50 and for DBP in the range of 0.19 to 0.39 , which was similar to the meta-analysis of Chen et al. [1] who found a mean correlation coefficient of 0.38 for SBP and of 0.28 for DBP.

One could ask whether BP tracking coefficients become larger among children with additional risk factors for hypertension, but in fact mathematically they are expected to remain similar or even become smaller, as verified with data from this study (results not shown). This happens since in subgroups with additional hypertension risk factors, BP values are more similar than in an unselected group and 

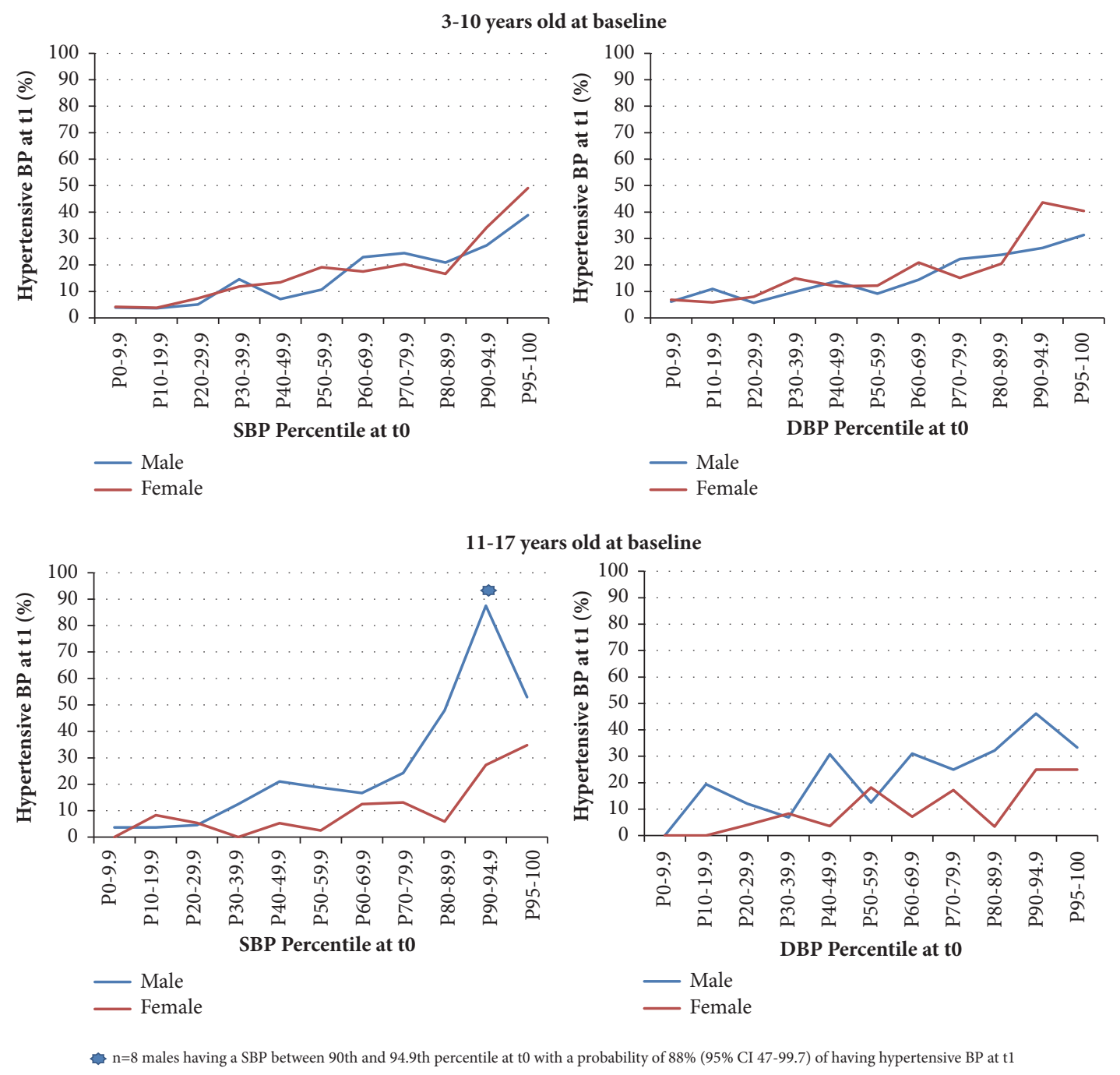

FIGURE 2: Probability of having hypertensive BP at six year follow-up (t1) according to baseline BP percentiles.

ranking to a greater extent due to chance. In other words, for combinations of risk factors, tracking coefficients are not an appropriate measure for assessing the predictability of future $\mathrm{BP}$ values.

Our results confirm previous findings that risk factors for hypertensive BP in young adulthood can be identified in childhood and adolescence $[2,17]$. However, the associations were moderate. After adjusting for various hypertension risk factors including overweight/obesity and parental hypertension, a child aged 3 to 10 years with hypertensive BP was more likely to have hypertensive BP six years later than a child in the same age group with a normal BP (OR 4.25, 95\% CI 2.90$6.23)$. For the age group 11 to 17 years the respective adjusted OR was 3.35 (95\% CI 1.60-7.01).

The association between overweight/obesity and hypertension in children was observed in several studies [18-20]. Our study showed that $36 \%$ of 3 - to 10 -year-old and $50 \%$ of 11- to 17 year-old obese children at baseline had a hypertensive $\mathrm{BP}$ at follow-up. The multivariable logistic regression analyses revealed that 3 - to 10 -year-old participants with a persistently or incidentally overweight/obesity were more likely to have a hypertensive BP at follow-up compared to participants with a persistently low or normal BMI trajectory, suggesting a potential action for prevention of future hypertension. In this regard, other studies have shown that children with higher BMI at follow-up changed BP standard deviation scores from initially normal to higher BP values [21]. Moreover, it has been observed that changes in BMI during growth predicted BP levels during adolescence [22]; for instance, the study of Kelly et al. [2] showed that the resolution of elevated BP in the transition from childhood to adulthood could be partially explained by improvements in associated factors such as by decreasing the BMI z-score between childhood and adulthood.

Our univariable logistic regression analysis showed that children with hypertensive parents were at higher risk of hypertension at follow-up compared to children without hypertensive parents. However, this was only confirmed in the multivariable analysis of 11-17-year olds. Children and adolescents with parental hypertension had an $18 \%$ and 
TABLE 4: Positive (PPV) and negative predictive value (NPV) of various hypertension risk factors or risk factor combinations for hypertensive $\mathrm{BP}^{*}$ at six-year follow-up.

\begin{tabular}{|c|c|c|c|c|}
\hline & $\mathrm{n}$ & Proportion at $\mathrm{t}_{0} \%(95 \% \mathrm{CI})$ & PPV \% (95\% CI) & NPV \% (95\% CI) \\
\hline \multicolumn{5}{|l|}{ Baseline age 3 to 10 years } \\
\hline Hypertensive $\mathrm{BP}$ at $\mathrm{t}_{0}$ & 2016 & $7.9(6.8-9.1)$ & $39(32-46)$ & $87(87-88)$ \\
\hline Overweight or obesity at $t_{0}$ & 2011 & $12(11-14)$ & $27(22-32)$ & $87(86-88)$ \\
\hline Obesity at $\mathrm{t}_{0}$ & 2011 & $2.2(1.7-3.0)$ & $36(23-50)$ & $86(85-86)$ \\
\hline Parental hypertension & 1942 & $29(27-31)$ & $18(15-20)$ & $87(86-88)$ \\
\hline Hypertensive BP and overweight or obesity at $t_{0}$ & 2011 & $2.0(1.5-2.7)$ & $48(33-62)$ & $86(86-86)$ \\
\hline Hypertensive $\mathrm{BP}$ and obesity at $\mathrm{t}_{0}$ & 2011 & $0.7(0.4-1.2)$ & $53(30-76)$ & $86(85-86)$ \\
\hline Hypertensive $\mathrm{BP}$ at $\mathrm{t}_{0}$ and parental hypertension & 1942 & $2.6(2.0-3.4)$ & $51(38-64)$ & $86(86-87)$ \\
\hline \multicolumn{5}{|l|}{ Baseline age 11 to 17 years } \\
\hline Hypertensive $\mathrm{BP}$ at $\mathrm{t}_{0}$ & 526 & $11(8.8-14)$ & $34(24-45)$ & $87(85-88)$ \\
\hline Overweight or obesity at $t_{0}$ & 525 & $17(14-20)$ & $24(17-33)$ & $87(85-88)$ \\
\hline Obesity at $\mathrm{t}_{0}$ & 525 & $2.7(1.6-4.5)$ & $50(27-74)$ & $86(85-87)$ \\
\hline Parental hypertension & 481 & $32(28-36)$ & $21(16-26)$ & $87(85-89)$ \\
\hline Hypertensive $\mathrm{BP}$ and overweight or obesity at $\mathrm{t}_{0}$ & 525 & $3.2(2.0-5.1)$ & $35(17-59)$ & $85(85-86)$ \\
\hline Hypertensive BP and obesity at $t_{0}$ & 525 & $0.6(0.2-1.8)$ & $\S$ & $85(85-85)$ \\
\hline Hypertensive $\mathrm{BP}$ at $\mathrm{t}_{0}$ and parental hypertension & 481 & $4.8(3.2-7.1)$ & $48(30-67)$ & $86(85-87)$ \\
\hline
\end{tabular}

${ }^{\S} \mathrm{n}<=10$ subjects

${ }^{*}$ Hypertensive BP: SBP or DBP at or above the 95th age-, sex-, and height-specific percentile for children and adolescents under 18 years or BP $\geq 140 / 90$ mmHg irrespective of the percentile; for adults aged 18 or older: $\geq 140 / 90 \mathrm{mmHg}$.

$21 \%$ probability of having a hypertensive BP at follow-up (Table 4). A familial predisposition for hypertension was reported in previous studies [23, 24]; furthermore, it has been evaluated whether BP screening should target only children with a parental hypertension instead of all children (universal screening), with the conclusion that this may not be a substantially better strategy [5].

In our data, obesity of the child/adolescent at $t_{0}$ was a significantly stronger predictor for hypertension at $t_{1}$ than parental hypertension in both age groups (Table 4).

Consistent with the literature [25-27] we observed male sex as a risk factor for hypertension in young adults: Figure 2 showed that 11- to 17-year-old males at baseline had a higher probability for having a hypertensive BP at follow-up (18 to 24 years) compared to females and Table 3 presented an OR $=0.33(95 \%$ CI $0.18-0.61)$ for 11- to 17 -year-old females at baseline, where they were less likely to have a hypertensive $\mathrm{BP}$ at follow-up compared to their male peers.

Contrary to other reports $[28,29]$, our study did not find a significant association between low birthweight and hypertension.

Even though we did not find significant associations between physical activity at $\mathrm{t}_{0}$ and hypertensive $\mathrm{BP}$ at follow-up, our estimates showed the tendency of a negative correlation between these two variables, which is in line with other studies [30, 31].

Our study does not intend to estimate the prevalence of hypertension in children, as the diagnosis of hypertension requires, among other things, BP measurements on several occasions; however, the proportion of children and adolescents with persistently elevated BP in our study was $3.1 \%$ for 3- to 10 -year olds at baseline and 3.8\% for 11- to 17 -year olds, which is in line with studies reporting prevalences of sustained hypertension in children and adolescents of $1 \%$ to $5 \%$ [32-34].

Major strengths of this study are the large national population database, coverage of a wide age range (3 to 17 years), the six-year follow-up, and standardized BP measurements according to the same protocol at baseline and follow-up examinations. However, despite using the same BP measurement devices, identical resting times, and the same measurement protocol, including performing BP measurements before motor performance testing, we cannot exclude the possibility that the environment and expectation of an extensive motor testing like in the MoMo study could have influenced BP at follow-up, leading to somewhat higher $\mathrm{BP}$ values and somewhat lower tracking indices. Another limitation is the possible selection bias arising from selective participation in the MoMo study and from longitudinal dropout. Sensitivity analyses with weights that partially account for this selection showed somewhat lower predictive values and thus did not alter the main conclusions. A further limitation is that the rule for KiGGS0 (2003-2006) was that the cuff should cover at least two-thirds of the upper arm length while current recommendations (2016 ESH guidelines [4]) suggest that the cuff size should cover $80-100 \%$ of the individual's arm circumference. Another limitation is that we had only two time points for assessment of the tracking; however, the KiGGS study just completed its second wave, allowing a future analysis of BP trajectories over a 10-year follow-up [35].

In conclusion, our analysis with recent population-based data from Germany shows that BP in children and adolescents tracks only moderately over six years; however, BP in childhood is the strongest independent predictor for future hypertensive BP measurements. 


\section{Data Availability}

The data used to support the findings of this study are available from the corresponding author upon request.

\section{Conflicts of Interest}

The authors declare that they have no conflicts of interest.

\section{Acknowledgments}

The authors thank the participants of the KiGGS and MoMo studies and all their colleagues who conducted and contributed with these studies: partial presentation of the manuscript (abstract) for DGEpi Congress 2017 in Gesundheitswesen 2017; 79(08/09): 656-804 DOI: 10.1055/s-0037-1605847. The study has been financed by the Robert Koch Institute, DZHK and the Institute for Sports and Sport Science.

\section{References}

[1] X. Chen and Y. Wang, "Tracking of blood pressure from childhood to adulthood: A systematic review and meta-regression analysis," Circulation, vol. 117, no. 25, pp. 3171-3180, 2008.

[2] R. K. Kelly, R. Thomson, K. J. Smith, T. Dwyer, A. Venn, and C. G. Magnussen, "Factors Affecting Tracking of Blood Pressure from Childhood to Adulthood: The Childhood Determinants of Adult Health Study," Journal of Pediatrics, vol. 167, no. 6, pp. 1422-1428, 2015.

[3] A. M. Toschke, L. Kohl, U. Mansmann, and R. Von Kries, "Meta-analysis of blood pressure tracking from childhood to adulthood and implications for the design of intervention trials," Acta Paediatrica, vol. 99, no. 1, pp. 24-29, 2010.

[4] E. Lurbe, E. Agabiti-Rosei, J. K. Cruickshank et al., "2016 European Society ofHypertension guidelines for themanagement of high blood pressure in children and adolescents," Journal of Hypertension, vol. 34, no. 10, pp. 1887-1920, 2016.

[5] C. Bloetzer, F. Paccaud, M. Burnier, P. Bovet, and A. Chiolero, "Performance of parental history for the targeted screening of hypertension in children," Journal of Hypertension, vol. 33, no. 6, pp. 1167-1173, 2015.

[6] B.-M. Kurth, P. Kamtsiuris, H. Hölling et al., "The challenge of comprehensively mapping children's health in a nation-wide health survey: Design of the German KiGGS-Study," $B M C$ Public Health, vol. 8, 2008.

[7] M. O. Wagner, K. Bös, D. Jekauc et al., "Cohort Profile: The Motorik-Modul Longitudinal Study: Physical fitness and physical activity as determinants of health development in German children and adolescents," International Journal of Epidemiology, vol. 43, no. 5, pp. 1410-1416, 2014.

[8] M. Lange, H. G. Butschalowsky, F. Jentsch et al., "The first KiGGS follow-up (KiGGS Wave 1): Study conduct, sample design, and response," Bundesgesundheitsblatt - Gesundheitsforschung - Gesundheitsschutz, vol. 57, no. 7, pp. 747-761, 2014.

[9] H. Neuhauser and M. Thamm, "Blood pressure measurement in the German Health Interview and Examination Survey for Children and Adolescents (KiGGS): Methodology and initial results," Bundesgesundheitsblatt - Gesundheitsforschung - Gesundheitsschutz, vol. 50, no. 5-6, pp. 728-735, 2007.

[10] A. Worth, A. Woll, C. Albrecht et al., MoMo-Längsschnittstudie , ,Physical Fitness and Physical Activity as Determinants of Health
Development in Children and Adolescents, Karlsruhe Germany: Karlsruher Institut für Technologie (KIT), 2015.

[11] H. K. Neuhauser, M. Thamm, U. Ellert, H. W. Hense, and A. S. Rosario, "Blood pressure percentiles by age and height from nonoverweight children and adolescents in Germany," Pediatrics, vol. 127, no. 4, pp. e978-e988, 2011.

[12] A. Hager, E. Wühl, G. Bönner, M. Hulpke-Wette, S. Läer, and J. Weil, S2k Leitlinie Pädiatrische Kardiologie, Pädiatrische Nephrologie und Pädiatrie: Arterielle Hypertonie: Deutsche Gesellschaft für Pädiatrische Kardiologie, 2015.

[13] T. J. Cole and T. Lobstein, "Extended international (IOTF) body mass index cut-offs for thinness, overweight and obesity," Pediatric Obesity, vol. 7, no. 4, pp. 284-294, 2012.

[14] K. Manz, S. Krug, A. Schienkiewitz, and J. D. Finger, "Determinants of organised sports participation patterns during the transition from childhood to adolescence in Germany: Results of a nationwide cohort study," BMC Public Health, vol. 16, no. 1, 2016.

[15] J. D. Finger, G. B. M. Mensink, W. Banzer, T. Lampert, and T. Tylleskär, "Physical activity, aerobic fitness and parental socioeconomic position among adolescents: The German Health Interview and Examination Survey for Children and Adolescents 2003-2006 (KiGGS)," International Journal of Behavioral Nutrition and Physical Activity, vol. 11, no. 1, article no. 43, 2014.

[16] B. Diedenhofen and J. Musch, "Cocor: A comprehensive solution for the statistical comparison of correlations," PLOS ONE, vol. 10, no. 4, 2015.

[17] R. M. Lauer and W. R. Clarke, "Childhood risk factors for high adult blood pressure: The Muscatine study," Pediatrics, vol. 84, no. 4, pp. 633-641, 1989.

[18] R. Din-Dzietham, Y. Liu, M.-V. Bielo, and F. Shamsa, "High blood pressure trends in children and adolescents in national surveys, 1963 to 2002," Circulation, vol. 116, no. 13, pp. 14881496, 2007.

[19] B. Falkner, S. S. Gidding, G. Ramirez-Garnica, S. A. Wiltrout, D. West, and E. B. Rappaport, "The relationship of body mass index and blood pressure in primary care pediatric patients," Journal of Pediatrics, vol. 148, no. 2, pp. 195-200, 2006.

[20] R.-C. Huang, S. Burrows, T. A. Mori, W. H. Oddy, and L. J. Beilin, "Lifecourse adiposity and blood pressure between birth and 17 years old," American Journal of Hypertension, vol. 28, no. 8, pp. 1056-1063, 2015.

[21] A. Miersch, M. Vogel, R. Gausche et al., "Blood pressure tracking in children and adolescents," Pediatric Nephrology, vol. 28, no. 12, pp. 2351-2359, 2013.

[22] A. Kollias, K. Pantsiotou, N. Karpettas, L. Roussias, and G. S. Stergiou, "Tracking of blood pressure from childhood to adolescence in a Greek cohort," European Journal of Public Health, vol. 22, no. 3, pp. 389-393, 2012.

[23] T. J. Niiranen, E. L. McCabe, M. G. Larson et al., "Risk for hypertension crosses generations in the community: Amulti-generational cohort study," European Heart Journal, vol. 38, no. 29, pp. 2300-2308, 2017.

[24] R. F. Theodore, J. Broadbent, D. Nagin et al., "Childhood to Early-Midlife Systolic Blood Pressure Trajectories: Early-Life Predictors, Effect Modifiers, and Adult Cardiovascular Outcomes," Hypertension, vol. 66, no. 6, pp. 1108-1115, 2015.

[25] H. K. Neuhauser, C. Adler, A. S. Rosario, C. Diederichs, and U. Ellert, "Hypertension prevalence, awareness, treatment and control in Germany 1998 and 2008-11," Journal of Human Hypertension, vol. 29, no. 4, pp. 247-253, 2015. 
[26] G. Sarganas and H. K. Neuhauser, "The persisting gender gap in hypertension management and control in Germany: 1998 and 2008-2011," Hypertension Research, vol. 39, no. 6, pp. 457-466, 2016.

[27] K. Wolf-Maier, R. S. Cooper, J. R. Banegas et al., "Hypertension prevalence and blood pressure levels in 6 European countries, Canada, and the United States," The Journal of the American Medical Association, vol. 289, no. 18, pp. 2363-2369, 2003.

[28] M. Gamborg, L. Byberg, F. Rasmussen et al., "Birth weight and systolic blood pressure in adolescence and adulthood: Metaregression analysis of sex- and age-specific results from 20 nordic studies," American Journal of Epidemiology, vol. 166, no. 6, pp. 634-645, 2007.

[29] M. Mu, S.-F. Wang, J. Sheng et al., "Birth weight and subsequent blood pressure: A meta-analysis," Archives of Cardiovascular Diseases, vol. 105, no. 2, pp. 99-113, 2012.

[30] L. B. Andersen, "Tracking of risk factors for coronary heart disease from adolescence to young adulthood with special emphasis on physical activity and fitness. A longitudinal study," Danish Medical Journal, vol. 43, no. 5, pp. 407-418, 1996.

[31] D. A. Jenner, R. Vandongen, and L. J. Beilin, "Relationships between blood pressure and measures of dietary energy intake, physical fitness, and physical activity in Australian children aged 11-12 years," Journal of Epidemiology and Community Health, vol. 46, no. 2, pp. 108-113, 1992.

[32] B. Falkner, "Hypertension in children and adolescents: epidemiology and natural history," Pediatric Nephrology, vol. 25, no. 7, pp. 1219-1224, 2010.

[33] A. R. Marras, P. P. Bassareo, and M. Ruscazio, "The prevalence of paediatric hypertension, emphasising the need to use specific population references: The Sardinian Hypertensive Adolescents Research Programme Study," Cardiology in the Young, vol. 19, no. 3, pp. 233-238, 2009.

[34] P. P. Bassareo and G. Mercuro, "Pediatric hypertension: An update on a burning problem," World Journal of Cardiology, vol. 6, no. 5, p. 253, 2014.

[35] E. Mauz, A. Gößwald, P. Kamtsiuris et al. et al., "New data for action. Data collection for KiGGS Wave 2 has been completed," Journal of Health Monitoring, vol. 2, pp. 2-27, 2017. 